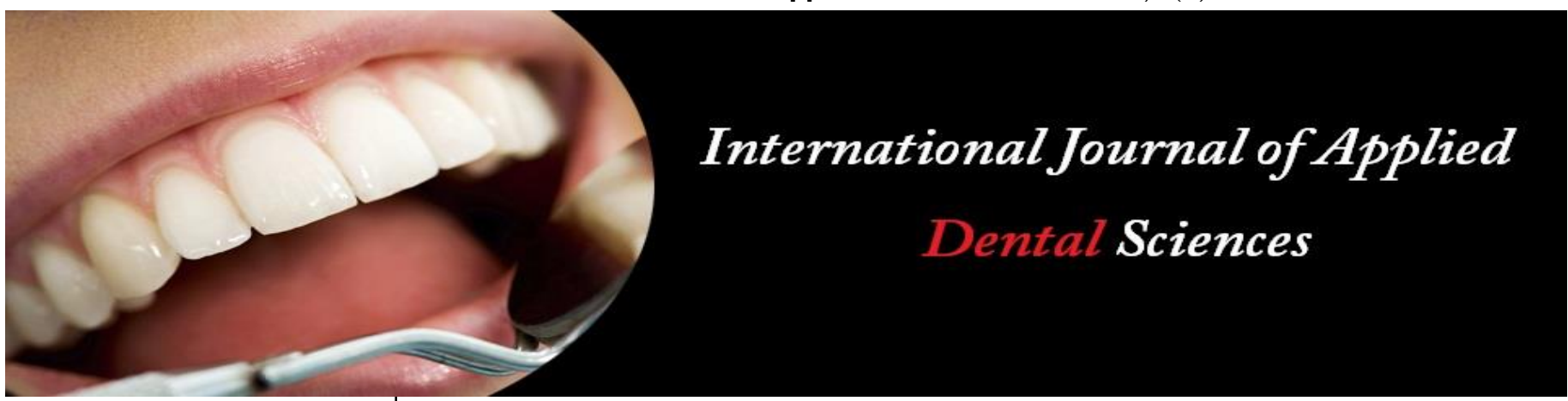

ISSN Print: $2394-7489$

ISSN Online: $2394-7497$

IJADS 2021; 7(4): 325-328

(C) 2021 IJADS

www.oraljournal.com

Received: 25-08-2021

Accepted: 27-09-2021

Dr. Nazima Jamal

MDS Orthodontics and Dentofacia Orthopedic, Srinagar, Jammu and Kashmir, India

Dr. Ausma Malik

MDS Orthodontics and Dentofacia Orthopedic, Srinagar, Jammu and Kashmir, India
Corresponding Author: Dr. Nazima Jamal

MDS Orthodontics and Dentofacial Orthopedic, Srinagar, Jammu and Kashmir, India

\section{Evaluation and comparison of shear bond strength of all surface bonding primer with a conventional primer using two different materials of brackets bonded to different surfaces: An in vitro study}

\author{
Dr. Nazima Jamal and Dr. Ausma Malik
}

DOI: $\underline{\text { https://doi.org/10.22271/oral.2021.v7.i4e.1391 }}$

\begin{abstract}
Orthodontic treatment frequently requires bonding brackets onto various types of surfaces like enamel, metal restorations, composites, ceramics, zirconia, acrylic temporaries etc. The development of new materials and techniques, simplifies the clinical procedures. The purpose of the present study was to evaluate the SBS of all surface bonding Primer (Assure plus Primer) compared with conventional primer (Transbond XT) onto tooth, acrylic temporary crown and Zirconia crown surfaces using metal and ceramic brackets. 120 samples were divided into 3 Groups - Tooth surfaces $(n=40)$, Acrylic temporary crown $(n=40)$ and PFM crown surfaces $(n=40)$. Subgroup A surfaces were primed with Transbond XT and bonded using Transbond XT adhesive paste to metal brackets and ceramic brackets. Subgroups B were primed with Assure Plus and bonded using Transbond XT adhesive paste. After debonding procedure, SBS was assessed. Group-III showed higher SBS values compared to Group-II and Group-I. The least SBS was seen in Group I, Subgroup-I B, Assure Plus Primer and metal brackets combination and the highest was seen in Group-III, Subgroup-III B, Assure Plus Primer and ceramic brackets combination.
\end{abstract}

Keywords: all surface bonding primer, acrylic temporary crowns, PFM crowns, shear bond strength

\section{Introduction}

The attachment of brackets to teeth plays a key role in the early stages of orthodontics treatment. Formerly, this was achieved by banding the teeth, but the introduction of the acidetching of enamel and the direct bonding of brackets has led to dramatic changes in the practice of orthodontics (Zachrisson, 1985) ${ }^{[1]}$. In recent times, Orthodontic treatment is being increasingly opted for by adults. Adult population inevitably present with multiple restored teeth and prosthesis due to missing or carious teeth. As many patients have teeth with various restorative materials, such as composite resin, amalgam, and porcelain, orthodontists are more likely to face difficulty in bonding orthodontic attachments to these materials ${ }^{[2]}$. Several authors have used different materials and adhesive techniques with the objective of improving the bonding durability and developing more suitable orthodontic attachments. Various authors have studied adhesive materials and different types of brackets commonly used in orthodontics in order to increase their adhesiveness to the enamel surface. One of the methods of increasing bracket bonding involves application of various primers to the bonding surface ${ }^{[3]}$. The aim of this study is to evaluate and compare shear bond strength of all surface bonding Primer with a conventional Primer using two different materials of brackets bonded to different surfaces.

\section{Material and Methods}

Materials used were non-carious human maxillary 1st premolar teeth, acrylic crowns (3M ESPE protemp 4, USA), Porcelain-Fused-to-Metal (PFM) crowns prepared on one extracted tooth (Vita, Germany) partially embedded in acrylic blocks but with buccal surfaces exposed. Each group had 40 specimens embedded in acrylic blocks. Two types of brackets were used 1) Metal brackets (MBT, 0.022" slot, Gemini series, 3M Unitek, USA) and 2) Ceramic brackets 
(MBT, 0.022" slot, Clarity series, 3M Unitek, USA). An invitro study was carried out. In group I 40 therapeutically extracted human maxillary $1^{\text {st }}$ premolar teeth were collected, cleaned with ultrasonic scaler, lightly pumiced and stored in distilled water at room temperature before use. Group-I $(n=40)$ was etched with $37 \%$ of Orthophosphoric acid for 15 seconds. The tooth surfaces were rinsed with water and dried. Group-I $(n=40)$ was further divided into Subgroup-I A (Primed with Transbond XT) and Subgroups-I B (Primed with Assure Plus). Subgroup-I A $(n=20)$ is further Sub-divided into those bonded to metal brackets $(n=10)$ and those bonded ceramic brackets $(n=10)$. Subgroup-I $B(n=20)$ is further subdivided into those bonded to metal brackets $(n=10)$ and those bonded to ceramic brackets $(n=10)$. For group II 40 acrylic crowns of the material supplied in automix cartridges was injected into silicon moulds replicating premolar tooth anatomy, set by chemical curing. The set restoration was removed and trimmed and polished with a pumice slurry. It was mounted on the acrylic blocks. Their surfaces were etched with $37 \%$ orthophosphoroc acid. The specimens were further divided into subgroups like group I. For group III crown preparation was done on one extracted premolar tooth and elastomeric impression was recorded and PFM crowns were fabricated. These PFM crowns were mounted in a similar way as mentioned above but buccal surfaces were exposed. The buccal surface of the crowns were roughened with a fine diamond bur, rinsed and dried. The sample were divided into Subgroup-III A and Subgroup-III B. In Subgroup-III A, PFM crowns were etched with $9 \%$ hydrofluoric acid as per manufacturer's instructions for 90 seconds and rinsed with water spray and air dried and then silane coupling agent (Ultra Dent ${ }^{\circledR}$ ) was applied on to the buccal surface for 60 seconds and air dried. A thin uniform coat of Transbond XT Primer was applied and light cured. The samples were subdivided into 2- one in which 10 metal brackets were bonded and another where 10 ceramic brackets were bonded using Transbond XT light cure adhesive paste and light cured as was mentioned in Group-I and Group-II. In Subgroup-III B, PFM crowns of roughened buccal surfaces, were primed one coat of Reliance Porcelain Conditioner for 60 seconds, and then air dried. One coat of Assure Plus
Primer was applied and lightly dried with air and light cured. The samples were divided into 2-one in which 10 metal brackets were bonded and another where 10 ceramic brackets were bonded. All the samples were stored in distilled water for one week at room temperature.

2.1 Debonding Procedure: The samples were positioned in the universal testing machine with the long axis parallel to the direction of the load application. A stainless steel wire 0.009" diameter in the shape of a loop was fixed to the upper cross head at one end and was adjusted to engage the bracket at the other end. A crosshead speed of $10 \mathrm{~mm} /$ minute was used and the maximum load necessary to debond the brackets was recorded. The force required to shear the bracket was recorded and the shear bond strength was calculated.

2.2 Statistical Analysis: It including the mean, standard deviation (SD), minimum and maximum values was calculated for all the groups of samples tested. Comparison of the means of SBS values was made with factorial Analysis of variance (ANOVA). The level of significance [p-value] was set at $p<0.05$. The results of the Shear Bond Strength was subjected to Weibull analysis (survival analysis) which facilitates comparison between the groups.

\section{Results}

The test results demonstrated that, the mean Shear Bond Strength of Metal bracket using Transbond XT Primer was $8.231 \pm 2.328$, whereas for ceramic bracket, it was $8.297 \pm$ 3.152. And the metal brackets using Assure plus primer, the mean Shear Bond Strength, was $15.077 \pm 6.701$, whereas for ceramic brackets, it was $17.297 \pm 3.033$. These differences in the mean Shear Bond Strength between different study Groups was Statistically Significant at $P<0.001$ for group III (PFM crown surface). (Table 1). Among the 3 Groups, the least Shear Bond Strength showed in Group I, Subgroup-IB, Assure Plus Primer and metal brackets combination (6.313 $\mathrm{MPa}$ ) and the highest was seen in Group-III, Subgroup-IIIB, Assure Plus Primer and ceramic brackets combination (17.297 $\mathrm{MPa})$.

Table 1: Comparison of Mean shear bond strength [MPa] between different groups based on the surfaces using one-way ANOVA test followed by Tukey's post hoc Analysis

\begin{tabular}{|c|c|c|c|c|c|c|c|c|c|}
\hline Surfaces & Groups & $\mathbf{N}$ & Mean & SD & Std. Error & Min & Max & $\mathbf{F}$ & P-Value \\
\hline \multirow{4}{*}{ Tooth } & MB_Transbond XT & 10 & 6.552 & 2.988 & 0.945 & 0.95 & 10.01 & \multirow{4}{*}{0.311} & \multirow{4}{*}{0.82} \\
\hline & CB_Transbond XT & 10 & 6.440 & 1.653 & 0.523 & 2.81 & 8.81 & & \\
\hline & MB_Assure plus & 10 & 6.313 & 3.101 & 0.981 & 1.3 & 10.82 & & \\
\hline & CB-_Assure plus & 10 & 7.296 & 1.938 & 0.613 & 4.74 & 11.05 & & \\
\hline \multirow{4}{*}{ Acrylic crown } & MB_Transbond XT & 10 & 9.138 & 4.668 & 1.476 & 1.84 & 15.29 & \multirow{4}{*}{1.422} & \multirow{4}{*}{0.25} \\
\hline & CB_Transbond XT & 10 & 10.552 & 2.863 & 0.905 & 6.06 & 14.74 & & \\
\hline & MB_Assure plus & 10 & 12.525 & 3.002 & 0.949 & 6.63 & 16.22 & & \\
\hline & CB_Assure plus & 10 & 10.384 & 4.022 & 1.272 & 3.64 & 15.61 & & \\
\hline \multirow{4}{*}{ PFM crown } & MB_Transbond XT & 10 & 8.231 & 2.328 & 0.736 & 5.19 & 11.86 & \multirow{4}{*}{12.523} & \multirow{4}{*}{$<0.001 *$} \\
\hline & CB_Transbond XT & 10 & 8.297 & 3.152 & 0.997 & 3.09 & 12.39 & & \\
\hline & MB_Assure plus & 10 & 15.077 & 6.701 & 2.119 & 6.05 & 25.69 & & \\
\hline & CB_Assure plus & 10 & 17.297 & 3.033 & 0.959 & 10.45 & 20.39 & & \\
\hline
\end{tabular}

\footnotetext{
* denotes Statistically Significant
}

Multiple comparison of the mean differences in the Shear Bond Strength between the Groups showed, that metal brackets with Transbond XT Primer showed Significantly lesser mean Shear Bond Strength as compared to Assure Plus Primer, for both metal \& ceramic brackets at $\mathrm{P}=0.004$ \& $P<0.001$ respectively. The ceramic brackets bonded with
Transbond XT Primer also showed similar differences with Assure Plus Primer, for both metal \& ceramic brackets at $\mathrm{P}=0.005^{*} \& P<0.001$ respectively. However, the mean differences between the metal \& ceramic brackets using the same primer, either Transbond XT or Assure plus did not show any Statistically Significant differences. (Table 2) 
Table 2: Multiple comparison of Mean Shear Bond Strength between Groups based on the surfaces using Tukey's HSD post hoc analysis.

\begin{tabular}{|c|c|c|c|c|c|c|}
\hline Surface & G1 Vs G2 & G1 Vs G3 & G1 Vs G4 & G2 Vs G3 & G2 Vs G4 & G3 Vs G4 \\
\hline Tooth & 1.00 & 1.00 & 0.91 & 1.00 & 0.87 & 0.82 \\
\hline Acrylic surface & 0.83 & 0.19 & 0.88 & 0.64 & 1.00 & 0.58 \\
\hline Ceramic surface & 1.00 & $0.004^{*}$ & $<0.001^{*}$ & $0.005^{*}$ & $<0.001^{*}$ & 0.64 \\
\hline
\end{tabular}

Weibull analysis was done to evaluate probability of failure of metal and ceramic brackets with different primers. (Table 3)

Table 3: Probability of failure

\begin{tabular}{|c|c|c|}
\hline Group I Tooth surface & N & Probability of failure \\
\hline MB- Transbond XT & 10 & $70 \%$ \\
\hline CB- Transbond XT & 10 & $90 \%$ \\
\hline MB- Assure Plus & 10 & $70 \%$ \\
\hline CB- Assure Plus & 10 & $80 \%$ \\
\hline Group II Acrylic surface & & \\
\hline MB- Transbond XT & 10 & $30 \%$ \\
\hline CB- Transbond XT & 10 & $20 \%$ \\
\hline MB- Assure Plus & 10 & $10 \%$ \\
\hline CB- Assure Plus & 10 & $20 \%$ \\
\hline Group III Ceramic surface & & \\
\hline MB- Transbond XT & 10 & $60 \%$ \\
\hline CB- Transbond XT & 10 & $30 \%$ \\
\hline MB- Assure Plus & 10 & $10 \%$ \\
\hline CB- Assure Plus & 10 & $0 \%$ \\
\hline
\end{tabular}

Weibull analysis showed maximum failure probability with probability with Group-III, CB-Assure Plus is 0\%. (Figure 1) Group-I, CB-Transbond XT is $90 \%$ and minimum failure

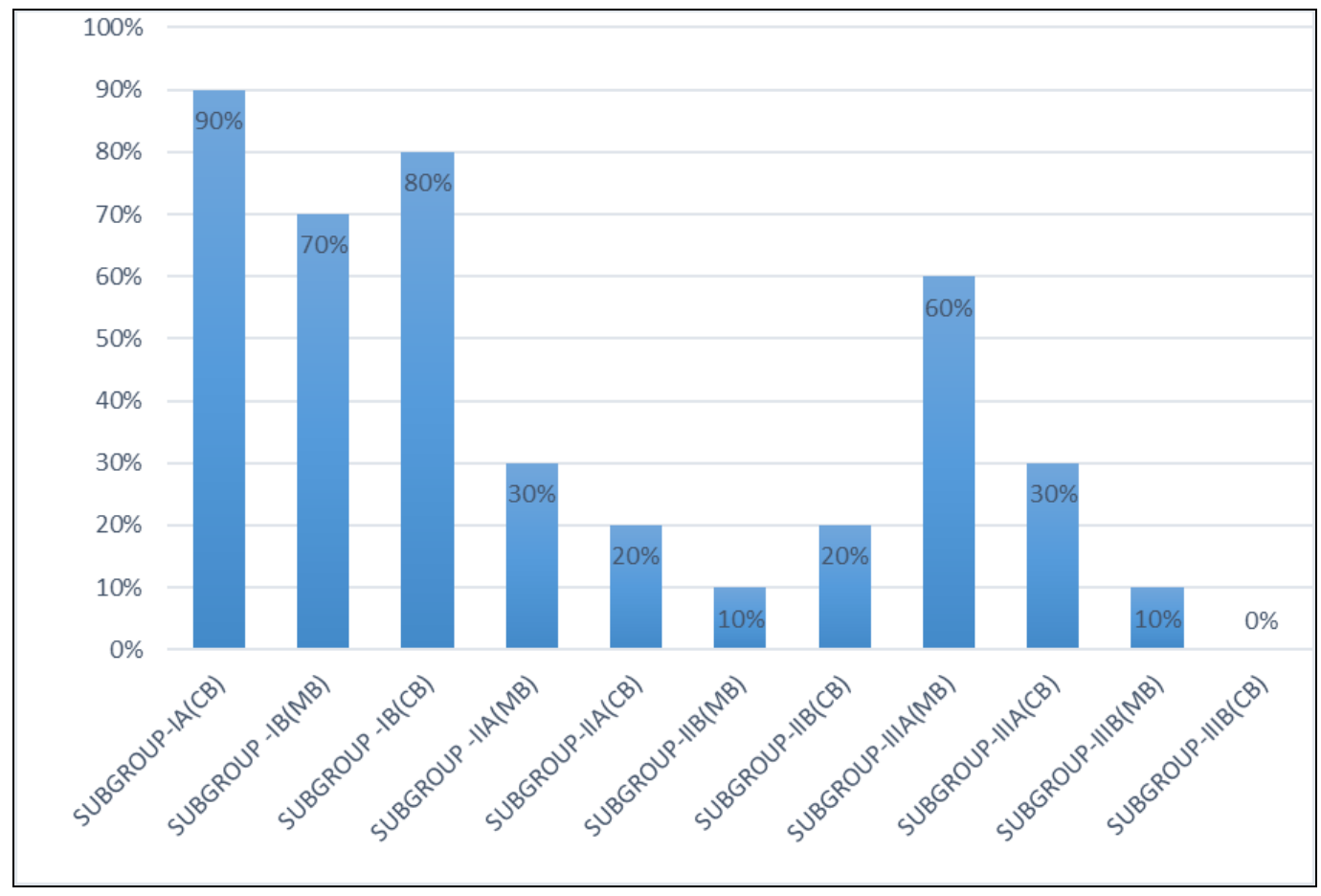

Fig 1: Probability of bond failure of different groups of brackets and primers used on different surfaces.

\section{Discussion}

Orthodontists are now approaching forty years of successful, reliable orthodontic bonding in offices around the world. The median bond failure rate for practitioners in the United States is presently around 5\%. The only teeth that were banded routinely by a majority of the U.S. orthodontists in a recent survey were the maxillary first molars, and all molars and premolars were banded less routinely than in the past ${ }^{[4]}$. Achieving a low bond failure rate had a high priority objective, since replacing loose brackets is inefficient, timeconsuming, and costly. Consequently, a continuous search is on for higher bond strengths, better adhesives, simpler procedure, and materials that will bond in the presence of saliva. However, most bond failures result from inconsistencies in the bonding technique and not because of the bonding resins, inadequate bond strengths, or quality of the brackets being used ${ }^{[5]}$. Bonding orthodontic attachments to the restoration surface is always challenging because of the high rate of bond failure during orthodontic treatment. Many researchers had suggested modified bonding procedures. Reynolds suggested that a minimum bond strength of 5.9 to 7.8 MPa was adequate for most clinical orthodontic needs ${ }^{[6]}$. It has been suggested that bond strength of 6-10 MPa are sufficient for orthodontic bonding ${ }^{[7]}$. As with any other 
indirectly fabricated restorations, the weakest part of the restorations is the bond between the resin luting agent layer and adjoining surfaces. In order to improve the bond strength between the luting agent and cured resin composite in restorative dentistry, surface treatment of indirect RCs with silane agent has been recommended. Bracket bonded to a fresh, roughened surface of an old composite restoration appear, in most instances, to be clinically successful. Yet, some authors recommend an intermediate primer as well, even though the bond is not between two dissimilar materials as in bonding to porcelain ${ }^{[8]}$. Three possible mechanisms during bracket bonding with the use of intermediate adhesive are chemical bond formation to the matrix, chemical bond to the exposed filler particles, and micromechanical retention caused by penetration of the monomer components to microcracks in the matrix. Clinically, the bonding between two composite layers was achieved in the presence of an oxygen-inhibited layer of unpolymerized resin ${ }^{[9]}$. Ceramic crowns are widely used to restore missing or damage teeth. Various types of ceramics has been developed, which may vary in chemical composition, method of manufacture, and physical properties. All-ceramic restorations, also known as 'metal free restoration' is the most recent types of ceramics. Ceramic is an inert material. It does not adhere chemically to any of the bonding resins. Conventional acid etching is ineffective in the preparation of ceramic surface for mechanical retention of brackets and orthodontic attachments. Thus, it is important to prepare ceramic surface adequately prior to bonding. Numerous approaches has been reported in the literature. They are classified into three major groups, namely mechanical, chemical, or a combination. Mechanical alteration of porcelain surfaces to increase bond strength has been achieved by sandblasting ${ }^{[10]}$. The chemical strength between the type of surface and adhesive, as well as the mechanical strength provided by the surface treatment, should be considered as intervening factors in the deficit adhesion to provisional acrylic surfaces ${ }^{[11]}$. While choosing the bonding material, some factors should be taken into consideration: resistance, longevity and removal of excess without damaging tooth surface. These factors can be evaluated in vitro by assessing the shear bond strength and ARI ${ }^{[12]}$.

\section{Conclusion}

The least Shear Bond Strength was seen in Group I, Subgroup-IB, Assure Plus Primer and metal brackets combination (6.313 Mand the highest was seen in Group-III, Subgroup-IIIB, Assure Plus Primer and ceramic brackets combination (17.297 MPa). The mean shear bond strength $(\mathrm{MPa})$ between different Groups showed that there were differences in Shear Bond Strength among three Groups, in which Group-III showed Statistically Significant $(<0.001)$ values and Group-I (0.82) and Group-II (0.25) showed Statistically Insignificant values. The values obtained were within the clinically acceptable levels and thus all three groups gave us clinically acceptable bond strengths.

\section{References}

1. Hsien Huang T, Tze Kao C. The shear bond strength of composite brackets on porcelain teeth. Eur J Orthod 2001;23(4):433-439.

2. Bayram M, Yesilyurt C, Kusgoz A, Ulker Mustafa, Metin Nur. Shear bond strength of orthodontic brackets to aged resin composite surface: effect of surface conditioning. Eur J Orthod. 2011;33(2):174-179.

3. Schmage et al. Influence of various surface-conditioning methods on the bond strength of metal brackets to ceramic surfaces. Am J Orthod Dentofacial Orthop. 2003;123(5):540-546.

4. Keim RG, Gottlieb EL, Nelson ALT, et al. JCO. Study of orthodontic diagnosis and treatment procedures, part I results and trends. J Clin Orthod. 2008;42(11):625-640.

5. Swartz ML. orthodontic bonding. Orthod select. 2004;16(2):1-4.

6. Reynolds I R. A review of direct orthodontic bonding. $\mathrm{Br}$ J Orthod 1975;2(3):171-178.

7. Buonocore MG. A simple method of increasing the adhesion of acrylic filling materials to enamel surface. $\mathrm{J}$ Dent Res. 1955;34(6):849-853.

8. Zachrisson BU, Buyukyilmaz T. Bonding in orthodontics. In: Graber T M, Vanarsdall R L, Vig K W L (eds). Orthodontics: current principles and techniques. Elsevier Inc., St Louis. 579-659.

9. Li J. Effect of surface properties on bond strength between layers of newly cured dental composites. J oral rehabil. 1997;24(5):358-360.

10. Zachrisson YO, Zachrisson BU, Buyukyilmaz T. Surface preparation for orthodontic bonding to porcelain. Am J Orthod Dentofacial Orthop. 1996;109(4):420-430.

11. Pinhoa MM, Mansob MC, Martind C, Júlio CM, Souzae $\mathrm{F}$, Almeidag RF et al. Adhesion strength of orthodontic brackets to acrylic surfaces. A systematic review on in vitro studies. Revista Portuguesa de Estomatologia e Cirurgia Maxilo-facial 2017;58(2).

12. Cochran D, O'Keefe KL, Turner DT, Powers JM. Bond strength of orthodontic composite cement to treated porcelain. Am J Orthod Dentofacial Orthop 1997;111(3):297-300. 